\title{
Expectativa e satisfação do acompanhamento pré-natal em gestantes de alto risco
}

\author{
Expectation and satisfaction of prenatal care in high-risk pregnant women
}

Expectativa y satisfacción de la atención prenatal em mujeres embarazadas de alto riesgo

Fabiana Fontana Medeiros ${ }^{1 *}$, Juliana Carvalho Lourenço ${ }^{1}$, Mariana Haddad Rodrigues ${ }^{1}$, Rosângela Aparecida Pimenta Ferrari ${ }^{1}$, Deise Serafim ${ }^{2}$, Alexandrina Aparecida Maciel Cardelli ${ }^{1}$.

\section{RESUMO}

Objetivo: Desvelar as representações sociais de mulheres em relação às expectativas e satisfação com o atendimento recebido no pré-natal de alto risco. Métodos: Trata-se de um estudo qualitativo, tendo a representação social como referencial teórico metodológico. A pesquisa foi realizada em um hospital filantrópico com puérperas que realizaram o pré-natal classificado de alto risco, em serviços de saúde público e privado. Foram realizadas 20 entrevistas entre os meses de outubro de 2017 e janeiro de 2018. As entrevistas foram gravadas e continham questões semiestruturadas para desvelar as expectativas e satisfação das mulheres com relação ao cuidado pré-natal que foi ofertado durante o período gravídico. $\mathrm{Na}$ análise dos dados adotou-se a análise de conteúdo. Resultados: A partir dos resultados das entrevistas emergiram duas categorias: "Desvelando a (in)satisfação com o atendimento pré-natal de alto risco em serviços de saúde e Expectativas das gestantes com o pré-natal de alto risco. O núcleo central foi desvelado pelas representações de acolhimento, segurança, esclarecimento de dúvidas, seguimento efetivo, preparação para as consultas e consulta com obstetra. Conclusão: Os cuidados e orientações recebidos durante o acompanhamento pré-natal de alto risco, foram satisfatórios em alguns pontos nos dois serviços de saúde, sendo atendido as expectativas das mulheres parcialmente.

Palavras-chave: Gravidez de alto risco, Cuidado pré-natal, Satisfação do usuário.

\begin{abstract}
Objective: To unveil womens's social representations in relation to expectations and satisfaction with the care received at high risk prenatal care. Methods: This is a qualitative study with social representation as the methodological theoretical framework. The research was conducted in a philanthopic hospital with puerperal women who underwent high-risk prenatal care at public and private health services. Twenty interviews were conducted between October 2017 and January 2018. The interviews were recorded and contained semistructured questions to unveil women's expectations and satisfaction regarding the prenatal care that was offered during the prenancy period. In the data analysis the content analysis was adopted. Results: From the interview results emerged two categories: "Unveiling (in) satisfaction with high-risk prenatal care in health services and Expectations of pregnant women with high-risk prenatal care. The central nucleus was unveiled by the representation of reception, security, clarification of doubts, effective follow-up, preparation for consultations and consultation with obstetricians. Conclusion: The care and guidance received during highrisk prenatal care were satisfactory at some points in both health services, and women's expectations were partially met.
\end{abstract}

Keywords: High-risk pregnancy, Prenatal care, User Satisfaction.

\footnotetext{
1 Universidade Estadual de Londrina (UEL), Londrina-Paraná. *E-mail: fontana.fabi@hotmail.com

2 Universidade Estadual de Maringá (UEM), Maringá-Paraná.
} 


\section{RESUMEN}

Objetivo: Desvelar las representaciones sociales de las mujeres en relación con las expectativas y la satisfacción con la atención recibida en la atención prenatal de alto riesgo. Métodos: Estudio cualitativo, con representación social como marco teórico metodológico. La investigación se realizó en un hospital filantrópico con madres que recibieron atención prenatal de alto riesgo en servicios de salud públicos y privados. Se realizaron veinte entrevistas entre octubre de 2017 y enero de 2018. Las entrevistas fueron grabadas y contenían preguntas semiestructuradas para revelar las expectativas y la satisfacción de las mujeres con respecto a la atención prenatal que se ofreció durante el período de embarazo. En el análisis de datos se adoptó el análisis de contenido. Resultados: De los resultados de la entrevista surgieron dos categorías: "Revelar (in) satisfacción con la atención prenatal de alto riesgo en los servicios de salud y Expectativas de las mujeres embarazadas con atención prenatal de alto riesgo. El núcleo central fue presentado por las representaciones de recepción, seguridad, aclaración de dudas, seguimiento efectivo, preparación para consultas y consulta con obstetras. Conclusión: La atención y la orientación recibidas durante la atención prenatal de alto riesgo fueron satisfactorias en algunos puntos en ambos servicios de salud, y las expectativas de las mujeres se cumplieron parcialmente.

Palabras clave: Embarazo de alto riesgo, Atención prenatal, Satisfacción del usuario.

\section{INTRODUÇÃO}

A gestação é um fenômeno fisiológico, sua evolução se dá na maior parte dos casos sem intercorrências. Porém, há uma parcela de gestantes que, por serem portadoras de alguma doença ou desenvolverem algum agravo, apresentam maior probabilidade de evolução desfavorável, tanto para o feto como para a mãe, essa parcela se constitui no grupo denominado "gestantes de alto risco". A intervenção precisa e precoce evita os retardos assistenciais capazes de gerar morbidade grave, morte materna ou perinatal (FEKADU M e REGASSA N, 2014).

A assistência pré-natal adequada colabora para prevenção da morbidade materna grave (MORAES APP, et al., 2013). O cuidado ofertado no acompanhamento à gestante pode prevenir inúmeros problemas maternos e neonatais, tendo como princípio a integralidade, ética e respeito, considerando um plano que atenda a real necessidade da mulher durante o período gestacional (CALOU CGP, et al., 2018).

A avaliação da qualidade dos serviços de saúde por meio de inquérito com os usuários difundiu-se a partir dos anos 1960 na Europa e Estados Unidos. No Brasil, a discussão sobre satisfação teve destaque na segunda metade da década de 1990, desde então, passou a ser valorizada como um indicador sensível, mas também da adesão ao tratamento, da relação profissional-paciente e da adequação do local da assistência (PAES NA, et al., 2014).

A satisfação do usuário destaca-se como indicador de qualidade na avaliação dos serviços de saúde, por meio da opinião dos usuários. O nível da referida reflete nos diversos momentos do atendimento. Neste contexto, o bom relacionamento entre profissionais e o paciente é a maior contribuição que se pode oferecer, com efeitos positivos no tratamento (JORGEA MSB, et al., 2007). Interliga-se a satisfação com o atendimento das expectativas da mulher e, essas, são conhecidas e trabalhadas a partir dos discursos apresentados por elas. A expectativa pode ser definida como a antecipação dos cuidados que ocorrem durante ou como resultado de saúde, sendo o que as pessoas esperam receber, e este pode ser influenciado pela maneira que irão avaliar os serviços recebidos (BOWLING A, et al., 2013).

Observa-se uma lacuna na avaliação da assistência gestacional de risco, a partir da satisfação e expectativa do atendimento prestado no serviço público e privado, os quais podem apontar parâmetros para a qualidade assistencial. Nesse sentido, a satisfação e expectativa com o atendimento recebido em mulheres que são acompanhadas no pré-natal de alto risco, podem servir de subsídios para a qualidade da assistência pré-natal. Este estudo teve como objetivo desvelar as representações sociais de mulheres em relação às expectativas e satisfação com o atendimento recebido no pré-natal de alto risco. 


\section{MÉTODOS}

Trata-se de uma pesquisa qualitativa, tendo como referencial teórico e metodológico a Teoria das Representações Sociais.O presente estudo foi realizado em uma maternidade de um hospital terciário e filantrópico do norte do Paraná, referência para gestação de alto risco na região. A coleta de dados ocorreu de outubro de 2017 a janeiro de 2018.

Como critérios de inclusão do estudo foram consideradas a classificação para gestação de alto risco, conforme Linha Guia Rede Mãe Paranaense (PARANÁ, 2018), assim como, as intercorrências clínicas da gestação atual, ter idade maior ou igual a dezoito anos, ter como produto de concepção recém-nascido vivo. E os critérios de exclusão foram: mãe sedada ou em ventilação mecânica. A população do estudo foi constituída de vinte puérperas que frequentaram o pré-natal de alto risco em serviços de atenção primária, secundária e privado, que tiveram como desfecho parto vaginal ou cesariana. As entrevistas ocorreram durante sua internação, e em momento oportuno definido pelas mesmas.

As participantes tiveram como desfecho de via de parto: sete partos vaginais e treze cesarianas. A coleta de dados foi realizada pela própria pesquisadora, com formação acadêmica nível de mestrado em obstetrícia. Realizou-se teste piloto a três puérperas, sendo adequado o formulário semiestruturado com questões socioeconômicas. Após, realizou-se entrevista tendo como questão norteadora: O que você esperava encontrar no seu pré-natal? Você foi atendida como esperava? O que você acredita que poderia ter sido diferente? Utilizou-se o critério de saturação dos dados.

A pesquisadora utilizou diário de campo para anotações diárias durante a coleta de dados. Cada entrevista teve, em média, duração de sessenta minutos. Utilizou-se gravador de voz e as entrevistas foram transcritas diariamente após a coleta de dados. Houve correção na transcrição do português e vícios de linguagem, não comprometendo o sentido das falas. Os dados foram analisados utilizando-se a análise de conteúdo de Bardin (BARDIN L, 2011).

Utilizou-se como codificação das falas (SUS) para as mulheres que realizaram seu acompanhamento gestacional no serviço público e (PRI) para as mulheres que realizaram seu pré-natal no serviço privado, seguido do número da entrevista, mantendo desta forma o sigilo das participantes. O estudo seguiu as recomendações éticas da resolução 466/2012, do Conselho Nacional de Saúde; o trabalho foi aprovado pelo Comitê de Ética em Pesquisa Envolvendo Seres Humanos da Universidade Estadual de Londrina (CEP/UEL) com o parecer nำ 2.323.783, CAAE: 78643417400005231.

\section{RESULTADOS}

A partir do pressuposto da teoria das representações sociais, analisou-se a percepção das gestantes de alto risco que frequentaram o pré-natal em serviços de saúde público e privado sobre as expectativas e satisfação das mulheres. Desvelou-se que a satisfação entre os serviços foram similares, sendo diferenciada por meio da organização dos mesmos, no serviço privado ocorreu maior disponibilidade quanto ao acesso aos profissionais. Por outro lado, a exclusividade da presença da enfermeira na atenção primária foi um diferencial para esse serviço, em que as atribuições dos profissionais são distribuídas conforme sua categoria.

Quanto ao perfil socioeconômico demográfico das participantes, o grupo etário mais prevalente com onze mulheres foi acima de trinta anos, variando entre dezoito e quarenta e quatro anos; treze eram casadas e possuíam ensino médio, e dez delas tinham algum tipo de ocupação. Emergiram duas categorias temáticas: "Desvelando a (in)satisfação com atendimento pré-natal de alto risco em serviços de saúde" e "Expectativas das gestantes com o pré-natal de alto risco".A partir das categorias foi desvelado o núcleo central pelas representações de acolhimento, esclarecimento de dúvidas, seguimento efetivo e consulta com médico obstetra.

\section{Desvelando a (in)satisfação com atendimento pré-natal de alto risco em serviços de saúde}

Houve satisfação quanto ao atendimento no pré-natal, evidenciada por periodicidade adequada na realização das consultas e esclarecimento de dúvidas. A periodicidade das consultas gerou satisfação quanto 
ao pré-natal, pois quando agendadas e realizadas conforme recomendação para cada trimestre gestacional, pode ter contribuído para o sentimento de segurança quanto à saúde materna e fetal.

"Eu gostei do atendimento. Uma vez por mês no começo. Depois começou duas, agora para o final (...). Quando eu tinha dúvida de alguma coisa, eu perguntava (SUS $1)^{\prime \prime}$.

“(...) Até mesmo nas frequências das consultas, tudo isso eu acredito que foi satisfatório. Até mesmo a respeito de algumas condutas que para mim foi novidade, ele sempre foi bastante esclarecedor, convincente (PRI 1)".

A orientação de preparar-se para consultas subsequentes, anotando dúvidas a serem esclarecidas futuramente, foi importante para gerar satisfação quanto ao atendimento, evidenciada pela abertura dos profissionais em demonstrar disponibilidade para responder aos questionamentos inerentes a esse período, permitindo estabelecer vínculo com abertura para que a gestante pudesse sentir-se à vontade nesta relação profissional-paciente.

"Superou porque eu não sabia que ia ser tão bem explicado, da maneira que foi (...) Ele falou assim, durante o intervalo de uma consulta para outra, você anota o que você tem dúvida, (...) não existe dúvida boba, dúvida é dúvida. Você chega aqui que a gente vai esclarecer sim (SUS 2)".

"Ah, eu tentava tirar todas as minhas dúvidas com o meu médico, eu sou muito curiosa, eu perguntava tudo com relação ao ultrassom, se estava tudo bem (...) para mim foi muito bom (PRI 2)".

O profissional demonstrou-se acessível, por meio do acolhimento evidenciado na oferta de cuidado integral com escuta ativa, para além da assistência obstétrica, abrangendo cuidados emocionais e psicológicos, como a continuidade de tratamento com medicações controladas, permitindo à mulher sentir-se cuidada de modo integral durante o seu pré-natal.

"(...) Ele se envolveu também na minha parte psicológica, ele se preocupou com isso, tanto é que eu estava... eu tinha perdido meu pai, ele perguntou se eu estava tomando medicação para depressão, ele não quis que eu diminuísse essa medicação. Porque eu queria diminuir por causa do bebê. Ele falou que não seria o certo, que o certo seria continuar. Então ele se envolveu praticamente quase no todo... ele foi um excelente obstetra (PRI 3).

"Ah! na verdade foi ótimo, porque lá eles são bem atenciosos, dão bastante atenção, e verificam se a gente está precisando de alguma coisa, vê se não está alterando o risco. Então para mim foi tudo muito bem (SUS 3)".

Consultas realizadas por enfermeira, destacaram-se quanto ao esclarecimento mais detalhado de todas as dúvidas, sendo percebidas como um diferencial do serviço público prestado quando comparado ao privado.

"Eu era bem atendida. Sim (...) tanto no público, quanto no privado. Eu gostei do tratamento que eu tive no público. Eu acho que eu tive mais detalhamento a respeito da gestação, no público. Assim, teve uma enfermeira que acho que era uma enfermeira formada em obstetrícia. Que sanava todas as dúvidas. Eu passei umas 2, 3 vezes com ela (PRI + SUS 4)".

Entretanto, observa-se insatisfação com o acompanhamento pré-natal desvelado pela dificuldade de acesso ao profissional obstetra, difícil acesso ao local de atendimento, percepção de não sentir-se acolhida, não ter suas dúvidas solucionadas, consultas com enfoque burocrático e tecnicista e não aderência ao serviço.

"Não (...) Ah, eu esperava mais atenção... E o povo lá não é muito atencioso com ninguém (...). Mais orientação. (...) eu moro muito longe, eu moro na entrada da cidade e o posto de saúde é no outro lado da cidade. Eu ia a pé, para voltar a pé (...). 
Poucas vezes encontrava o médico. Muitas vezes eu ligava para saber se a médica estava ou não estava, às vezes, eles falavam que estava e eu perdia a caminhada e chegava lá e não estava. Passava pelo clínico, mas muitas vezes voltava com dúvidas (SUS 5)".

A insatisfação foi gerada pelo fato de os procedimentos serem realizados rotineiramente, tal qual uma "linha de produção", no qual um profissional que não era da área da saúde realizava a técnica de pesagem, checagem e entrega de guias do convênio, demonstrando que o enfoque era os procedimentos em detrimento do acolhimento e cuidado.

"Não [risos]. No pré-natal? A gente só pesava, as recepcionistas lá me pesavam, entrava, chamava, olhava a guia, entregava a guia, olhava o exame, está tudo beleza, beleza? la embora, tchau e bênção, até na outra consulta. Esperava um pouco melhor. Mais atencioso (PRI 5)".

\section{Expectativas das gestantes com o pré-natal de alto risco}

Quanto às expectativas no pré-natal de alto risco, revelou-se a preocupação do bem-estar fetal, com necessidade da realização de exames de imagem e rotina, desvelando a preocupação da gestante com o feto e deixando suas necessidades de cuidado em segundo plano.

"Do pré-natal? (...) Ah, era mais os ultrassom, pra ver como o neném estava. $A$ expectativa era na ultrassom. $E$ dos exames também, de saber se estava tudo bem com o bebê, tudo mais (...) (PRI 6).

Ah, rotina, só para ver como o nenê estava. Para ver se estava crescendo, evoluindo (...). Era encontrar isso (SUS 6)".

A vitalidade fetal também esteve relacionada com o esclarecimento de dúvidas e acolhimento. As mulheres tinham expectativas semelhantes nos dois tipos de serviços, principalmente na questão relacionada ao parto.

O recebimento de informação sobre o tipo de parto durante o decorrer do pré-natal pode significar para a mulher diminuição de sua ansiedade. Compreende-se que a gestação com seguimento para o alto risco, pode inviabilizar determinada via de parto, entretanto é importante que o profissional pré-natalista direcione a consulta para possibilidade das vias de parto em tempo oportuno à necessidade da mulher, tendo em vista que $\mathrm{o}$ adiamento de informação pode ocasionar em insegurança à gestante.

"Eu acho que os médicos demoram um pouco para falar sobre o parto, tipo assim, "ah, eu vou esperar passar tantos meses para poder falar". Eu acho que tinha que ser uma das primeiras informações, porque você está correndo risco de ganhar com 5, $\operatorname{com}$ 6, $\operatorname{com} 7, \operatorname{com} 8, \operatorname{com}$ 9, então (...) (PRI 7)".

"Eu acho que (...) a explicação do parto e também do nascimento. Que assim, eu não sabia também que a criança podia ter também infecção urinária por uma bolsa ter rompido (...) Como ela teve (SUS 7)".

A não realização da consulta no ambulatório de alto risco, por falta de vagas, fez com que a gestante se sentisse fragilizada e prejudicada, pois tinha expectativa que poderia ter sido diferente o desfecho da sua gravidez, caso esse atendimento tivesse ocorrido em momento oportuno.

"Olha, o que eu senti falta foi do último pré-natal lá no Ambulatório de alto risco, porque estava com falta de vaga. Que a doutora já estava (...) cobrindo férias de outra profissional, e não tinha vaga para eu passar. Então na última semana, eu tive muitas contrações (...) tive hemorragia. Então, talvez, se eu tivesse passado com ela, se tivesse diagnosticado alguma coisa, talvez eu não teria passado por essa hemorragia, não sei? Então eu senti falta disso, porque durante o pré-natal dos outros meses fui bem atendida, sem nenhum problema (SUS 8)". 
$\mathrm{O}$ acolhimento oferecido à gestante de alto risco pode ocasionar no alcance de suas expectativas. A mulher sente-se segura com os cuidados direcionados à sua necessidade. Por outro lado, percebe-se que quando o acolhimento não é estabelecido no seguimento pré-natal, com a escuta eficaz, a mulher sente-se desorientada, não atingindo a expectativa desejada.

"Ah, eu acho que mais diálogo. Ter mais diálogo, as médicas terem mais paciência (...) mais paciência na hora da consulta (SUS 9).

O doutor sempre foi bem acolhedor comigo (...). Com relação a prematuridade, ele sempre deixou isso claro, que o risco sempre existia. Então, o cuidado durante toda a gestação foi bem redobrado, ainda mais por conta do histórico anterior, da perda anterior (PRI 1)".

O atendimento realizado com o clínico geral na assistência ao pré-natal de alto risco fez com que a mulher não se sentisse cuidada. Pode-se inferir que quando a assistência pré-natal ocorre com a aplicabilidade do cuidado integral, a mulher sente-se assistida.

"Ah, muitas das vezes eu chegava e a médica não estava. Aí eu tinha que passar por um clínico, já não era a mesma coisa, eu já voltava embora revoltada. Passava pelo clínico e ia embora com dor. Uma consulta geral (...), lá é assim. Passava pela médica e depois levava a carteirinha para as enfermeiras anotarem lá umas coisas (SUS 5)."

\section{DISCUSSÃO}

A avaliação da expectativa e satisfação das gestantes com relação às informações e cuidados recebidos durante o pré-natal, na percepção das puérperas, é uma importante estratégia de avaliação da qualidade dos serviços, partindo do pressuposto que a assistência deve ser centrada nas necessidades da mulher, porém no Brasil essa temática ainda é incipiente. A teoria da representação social permite reconhecer e valorizar o aspecto cognitivo do indivíduo, desvelando sua dimensão, que segundo esta perspectiva interfere nas práticas sociais, nas atitudes e condutas relativas ao objeto da representação. O conhecimento dos participantes para compreender o seu cotidiano e esclarecer o processo de assimilação dos fatos por meio da comunicação e comportamentos são focos dessa atenção (SILVA SED, et al., 2011).

As expectativas das pacientes são definidas como a antecipação dos cuidados susceptíveis a ocorrerem durante $o$ atendimento, sendo o que as pessoas esperam receber, e este pode ser influenciado pela maneira que irão avaliar os cuidados recebidos, que seria a satisfação com o esse atendimento (BOWLING A, et al., 2013). No presente estudo a satisfação foi referida nos dois tipos de serviços quanto à periodicidade das consultas, à disponibilidade dos profissionais e ao cuidado integral. Porém, a insatisfação foi desvelada em algumas falas quanto à falta do médico obstetra no serviço público e a falta de acolhimento como uma linha de produção no privado. Esses resultados diferem de um estudo realizado na rede hospitalar, na cidade do Recife, Pernambuco, onde a avaliação geral com o atendimento recebido apresentou diferenças entre os serviços, sendo maior a satisfação das usuárias com o atendimento recebido no hospital filantrópico e apresentaram insatisfação acima da esperada às gestantes assistidas nas unidades municipais e estaduais (SILVA ALA, et al., 2017).

Em uma pesquisa realizada no Rio Grande do Sul, na cidade de Porto Alegre, com mães entrevistadas na maternidade por ocasião do parto, foi evidenciado que gestantes atendidas no setor público estiveram em desvantagem em relação àquelas atendidas no setor privado. No que diz respeito à prevalência de realização de um determinado procedimento clínico, exame laboratorial ou orientação recebida durante a consulta de pré-natal (CÉSAR JA, et al., 2012). Na presente pesquisa, o setor público esteve em desvantagem com a questão do agendamento com seguimento de alto risco, impossibilitando o acesso às informações pertinentes ao seu risco. Por outo lado, o serviço privado não tem serviço específico para esse tipo de atendimento.

Nos estudos de Galle A, et al. (2015), identificou-se que a satisfação geral com os cuidados recebidos durante o pré-natal foi elevada, no entanto, as gestantes revelaram insatisfação em relação às informações 
recebidas durante as consultas, bem como os aspectos organizacionais dos cuidados pré-natais, demonstrando a necessidade de maiores esforços por parte dos profissionais de saúde envolvidos no acompanhamento dessas mulheres.

Desta forma vale ressaltar que a informação não depende do tipo de serviço que a mulher será acompanhada durante o pré-natal, mas do profissional que irá assisti-la durante este período, seguindo protocolos específicos para cada fase, assistindo a gestante na sua integralidade, com acolhimento, e respeito e orientações pertinentes a este momento. Neste sentido, Viellas EF, et al., (2014) descrevem que a satisfação, acolhimento e a manutenção do vínculo são primordiais para avaliar com maior eficácia a qualidade da assistência prestada. Neste estudo desvelou-se que a consulta com a enfermeira gerou melhor esclarecimento de dúvida e satisfação com atendimento recebido.

Em um estudo realizado em Belo Horizonte com gestantes de alto risco que frequentaram consultas de enfermagem, evidenciou-se oferta de espaço privilegiado para as narrativas, ambiência protetora, e contribuição para o alívio de sofrimento em determinados momentos, aumentando desta forma o vínculo entre paciente e profissional (ERRICO LSP, et al., 2018). No presente estudo, a vulnerabilidade no acompanhamento pré-natal, esteve presente, principalmente por não saberem que tipo de assistência deveria receber. Desta forma, não questionavam e aceitavam tudo que era imposto pelo médico, principalmente a questão relacionada à decisão sobre a via de parto.

Em uma revisão integrativa, onde foram analisados 17 estudos nacionais e internacionais, de 2000 a 2009 , sobre a percepção do parto vaginal e cesárea pelas puérperas, os fatores associados à maior satisfação foram a presença de acompanhante, o suporte emocional, as orientações no pré-natal, a qualidade na relação entre profissionais e gestantes, as informações durante a assistência e maior participação das mulheres no controle decisório (VELHO MB, et al., 2012). Em relação à localização geográfica, algumas gestantes tiveram dificuldade de acesso ao pré-natal, pois os serviços eram distantes de suas residências, dificultando a frequência nas consultas, fato este também observado em um estudo realizado na região metropolitana da grande Vitória (ESPOSTI CDD, et al., 2015).

Segundo as diretrizes do Programa de humanização do pré-natal e nascimento (PHPN), a proximidade geográfica da unidade de saúde da casa da gestante constitui-se num critério para determinar o local de realização do acompanhamento de pré-natal (BRASIL, 2000). Em um estudo realizado no interior do nordeste do Brasil sobre acesso e o acolhimento no cuidado pré-natal, descreve que a comunicação pautada na humanização e no acolhimento colabora para que a gestante mantenha vínculo com os serviços de saúde durante todo o período gestacional, reduzindo os riscos de intercorrências obstétricas. A comunicação e a interação de profissionais e gestantes evidenciaram satisfação da assistência prestada, conforme relatos das mulheres (SILVA MZN, et al., 2014). O núcleo central das representações construídas pelas mulheres deste estudo, como acolhimento, esclarecimento de dúvidas, seguimento efetivo e consulta com obstetra não difere dos achados de outras pesquisas, fato este que se torna bastante significativo em relação ao objeto em estudo.

\section{CONCLUSÃO}

Ao analisar os cuidados e orientações recebidos durante o pré-natal, estes foram satisfatórios, atendendo em alguns pontos as expectativas das mulheres. O que difere entre os estabelecimentos públicos e privados, é que cada tipo de serviço tem seus pontos fortes e fracos e seus processos de atendimento são desenhados de forma diferentes, embora o resultado final deva ser o mesmo, seguindo sempre os mesmos critérios de atenção. Como limitação do presente estudo destaca-se a abordagem a um grupo específico de mulheres, e sua realização ser em uma única instituição de saúde, entretanto o presente estudo apresenta dados valiosos para compreensão do fenômeno estudado.

\section{AGRADECIMENTOS}

À Coordenação de Aperfeiçoamento de Pessoal de Nível Superior (CAPES) pelo recebimento de bolsa de doutorado, número do processo 1779031 à autora Fabiana Fontana Medeiros. 


\section{REFERÊNCIAS}

1. BARDIN L. Análise de conteúdo. Lisboa: Editora Austin; 2011; 123p.

2. BOWLING A, et al. Patients' experiences of their healthcare in relation to their expectations and satisfaction: a population survey. J. R. Soc. Med., 2013; 106 (4): 143-149.

3. BRASIL. Ministério da Saúde. Assistência pré-natal: manual técnico. 3. ed. Brasília, DF, 2000.

4. CALOU CGP, et al. Maternal predictors related to quality of life in pregnant women in the Northeast of Brazil. Health Qual Life Outcomes, 2018; 16 (109).

5. CÉSAR JA, et al. Assistência pré-natal nos serviços públicos e privados de saúde: estudo transversal de base populacional em Rio Grande, Rio Grande do Sul, Brasil. Cad. Saúde Pública, 2012; 28 (11): 2106-2114.

6. ERRICO LSP, et al. O trabalho do enfermeiro no pré-natal de alto risco sob a ótica das necessidades humanas básicas. Rev. Bras. Enferm., 2018, 71 (3): 1257-1264.

7. ESPOSTI CDD, et al. Representações sociais sobre o acesso e o cuidado pré-natal no Sistema Único de Saúde da Região Metropolitana da Grande Vitória, Espírito Santo. Saude Soc., 2015, 24 (3): 765-779.

8. FEKADU M, REGASSA N. Skilled Delivery Care Service Utilization in Ethiopia: Analysis of Rural-Urban Differentials Based on National Demographic and Health Survey (DHS) Data. Afr. Health Sci., 2014, 14 (4): 974-984.

9. GALLE A, et al. Expectations and satisfaction with antenatal care among pregnant women with a focus on vulnerable groups: a descriptive study in Ghent. BMC Womens Health, 2015, 15 (1).

10. JORGEA MSB, et al. Avaliação da qualidade do programa saúde da família no ceará: a satisfação dos usuários. Rev. Baiana Saúde Pública, 2007, 31 (2): 256-266.

11. MORAES APP, et al. Morbidade materna grave: um estudo de caso-controle no Maranhão, Brasil. Reprod. Health, 2013, 10 (11).

12. PAES NA, et al. Satisfação dos usuários hipertensos com os serviços da rede de atenção primária no Brasil: um estudo de validação. Rev. Panam. Salud. Publica, 2014, 36 (2): 87-93.

13. PARANÁ. Secretaria de Estado da Saúde. Superintendência de Atenção à Saúde. Linha Guia Rede Mãe Paranaense. Curitiba, 2018.

14. SILVA ALA, et al. A qualidade do atendimento ao parto na rede pública hospitalar em uma capital brasileira: a satisfação das gestantes. Cad. Saúde Pública, 2017, 33 (12).

15. SILVA SED, et al. A teoria das representações sociais nas pesquisas da enfermagem brasileira. Rev. Bras. Enferm., 2011, 64 (5): 947-951.

16. SILVA MZN, et al. Acesso e acolhimento no cuidado pré-natal à luz de experiências de gestantes na Atenção Básica. Saúde em Debate, 2014, 38 (103).

17. VELHO MB, et al. Vivência do parto normal ou cesáreo: revisão integrativa sobre a percepção de mulheres. Texto Contexto Enferm., 2012, 21 (2): 458-466.

18. VIELLAS EF, et al. Assistência pré-natal no Brasil. Cad. Saúde Pública, 2014, 30 (1). 